\title{
Robotic Surgery in Otolaryngology-Head and Neck Surgery: Yesterday, Today and Tomorrow
}

\author{
Ali Bayram¹, Görkem Eskiizmir², Cemal Cingii ${ }^{3}$, Ehab Hanna ${ }^{4}$ \\ 1 Department of Otorhinolaryngology, Kayseri City Training and Research Hospital, Kayseri, Turkey. \\ 2 Department of Otorhinolaryngology, Celal Bayar University School of Medicine, Manisa, Turkey. \\ 3 Department of Otorhinolaryngology, Eskisehir Osmangazi University, Medical Faculty, Eskisehir, Turkey \\ 4 Department of Head and Neck Surgery, Center Medical Directory, Head and Neck Center, University of Texas, MD Anderson Cancer \\ Center, Houston, Texas. \\ Ali Bayram, ORCID: 0000-0002-0061-1755 \\ Görkem Eskiizmir, ORCID: 0000-0002-3125-8288 \\ Cemal Cingi, ORCID: 0000-0002-3125-8288 \\ Ehab Hanna, ORCID: 0000-0003-3241-2440
}

\begin{abstract}
In the present review, we aim to give an overview of the development and utilization of robotic surgery in otolaryngology-head and neck surgery from its introduction, current status, and future applications. Robotic surgery has been applied either pre-clinically or is already in clinical use for several indications in otolaryngology and head and neck surgery. Due to its rapid progress and technological development, it appears it will be a more active tool in the future for the diagnosis and treatment of a number of diseases in otolaryngology-head and neck surgery practice.
\end{abstract}

Keywords: Robotic surgical procedures, otolaryngology, head and neck surgery.

Correspondence: Ali Bayram

Department of Otorhinolaryngology, Kayseri City Training and Research Hospital, Kayseri, Turkey.

E-mail: dralibayram@gmail.com
Online available at: www.entupdatesjournal.org 


\section{Introduction}

Robotic surgery is an electromechanically-aided surgery that provides more efficiency and precision to the surgeon through high-definition (HD) three-dimensional visualization, precise fine-motor movements, and full wristed articulation. ${ }^{[1,2]}$ Robotic systems are not subject to the physiological tremor of human hands and provide the ability to perform effective surgery in tight anatomical confines. As an anatomical region with numerous limited-access and narrow anatomical spaces, the otolaryngology-head and neck region has been readily adopted to robotic surgeries involving benign and malignant lesions of the oropharynx, hypopharynx and larynx, microvascular reconstruction, thyroid and parathyroid diseases, obstructive sleep apnea (OSA), and sinonasal and skull base pathologies. ${ }^{[3-10]}$

\section{History of robotic surgery and current robotic platforms}

The emergence of robotic systems in human life was initially motivated by their deployment in hazardous industrial environments. From there, robotic technologies have evolved as a computer technology applied in medicine ${ }^{[1]}$ Although the interest of NASA for performing remote surgery on astronauts initially appeared in the 1970s (telepresence surgery), the first surgical robot, named "Arthrobot", was designed by the medical team of Dr. James McEwen and Geof Auchinlek in collaboration with the orthopedic surgeon Dr. Brian Dayand in 1983 and was used to assist orthopedic procedures in Canada. ${ }^{[12]}$ Telepresence represents the term defining sensations to give the appearance of being present at a place other than the real location, which was the underlying highly-sophisticated principle of utilizing robots in surgery. ${ }^{[7]}$ In 1993, Computer Motion (Santa Barbara, California, USA), a leading supplier of medical robots founded in 1989, introduced AESOP ${ }^{\circledR}$ (Automated Endoscope System for Optimal Positioning), which had a robotic arm to assist in laparoscopic surgery. $\mathrm{AESOP}^{\circledR}$ represented the first telepresence surgical robot approved by the Food and Drug Administration (FDA), in 1994. ${ }^{[13]}$ In 1998, the ZEUS $^{\circledast}$ robotic surgical system (Computer Motion, Inc.), the follow-up of AESOP, carried out the first full endoscopic robotic procedure, fallopian tube re-anastomosis, in Cleveland, USA. ${ }^{[14]}$ In 2001, a remote laparoscopic cholecystectomy in a 68-year-old female was performed by surgeons in New York and a patient in Strasbourg, France with the ZEUS ${ }^{\circledast}$ system, consisting of "surgeon-side" and "patient-side" connected through a high-speed terrestrial optical-fiber network (FranceTelecom/Equant). ${ }^{[15]}$

Intuitive Surgical (Sunnyvale, CA, USA), founded in 1995, launched the first (standard) da Vinci Surgical System in 1999. The name "da Vinci" was dedicated to Leonardo da Vinci, an Italian polymath of the Renaissance, who possibly designed the first known robot around the year 1495, Mechanical Knight. ${ }^{[16]}$ The da Vinci surgical system mainly includes two parts: (i) a surgeon console where the surgeon sits and performs surgery, and (ii) a patient-side cart where the patient is positioned during surgery, including three robotic arms that carry out the surgeon's commands, instruments with a specific surgical mission such as clamping, suturing, and tissue manipulation, and a vision system. In 2000, the da Vinci surgical system gained FDA approval for general laparoscopic surgery. ${ }^{[17]}$ In 2003, Computer Motion and Intuitive Surgical merged into a single company and the ZEUS ${ }^{\circledR}$ system was phased out in favor of the da Vinci system, with the da Vinci standard upgraded by the addition of a fourth robotic arm in the same year. In 2006, the da Vinci S system added HD vision and a multi-image display feature (TilePro $\left.{ }^{\circledR}\right)$. In 2009, the next model, da Vinci Si provided dual-console capability enabling collaborative surgical opportunities, improved $3 \mathrm{D}$ resolution, multi-source screens, and integrated control systems.. The Xi model of the da Vinci surgical robot released in 2014 hoped to address the shortcomings of da Vinci Si and was offered as an advanced tool for minimally invasive surgery with its novel overhead architecture, slimmer boom-mounted arms, improved motion range, guided targeting, and integrated auxiliary technology. The da Vinci single port (SP) model represents the company's $4^{\text {th }}$ generation robot, which has three articulating instrument arms and a single fully-wristed 3D-HD camera all deployed through a single 25 -mm port. ${ }^{[11]}$ In 2018, The FDA approved the marketed version of the da Vinci SP model for urological surgical procedures, which is suitable for a SP approach, and recently in 2019, the FDA cleared the marketed version for transoral surgeries in the oropharynx, restricted to benign tumors and malignant tumors that are classified as $\mathrm{T} 1$ and $\mathrm{T} 2$ in adults. ${ }^{[18]}$

Due to the growing interest in robotic surgery in the otolaryngology-head and neck surgery, several manufacturers introduced their technology in that space. Besides the da Vinci robotic systems, there are several commercially available robotic platforms on the market. Among these robotic platforms, there is the Flex ${ }^{\circledR}$ robotic system 
(Medrobotics Inc, Raynham, MA), which is composed of a hybrid system enabling endoscopic surgery with a robotically-driven scope, specifically designed for the head and neck region. The system provides robot-assisted visualization and surgical site access to the oropharynx, larynx, and hypopharynx and supports flexible instruments and surgical cutting devices through its accessory channels. In 2012, Rivera-Serrano et al. ${ }^{[19]}$ investigated the feasibility of visualizing the endolarynx transorally with a highly flexible robot without performing suspension of the larynx on two fresh and four preserved human specimens and achieved visualization of the endolarynx with standard mouth retractors. In 2015, Remacle et al. ${ }^{[20]}$ performed the first transoral robotic surgery (TORS) procedures on three patients with the Flex ${ }^{\circledR}$ robotic system and reported that the system offered good visualization of the surgical field without compromising safety or success. After subsequent human studies conducted in the head neck area, ${ }^{[21,22]}$ the system gained FDA approval in July 2015. ${ }^{[23]}$ In a recent article, Hussain et al. ${ }^{[2]}$ reported preliminary oncologic outcomes of 19 patients who underwent TORS with the Flex ${ }^{\circledR}$ robotic system for supraglottic carcinoma and concluded that the system could potentially be a valuable addition to existing surgical modalities. However, it is noteworthy that the Flex ${ }^{\circledR}$ robotic system is currently under development, and further studies are required to determine its use in different otolaryngological procedures.

Currently, there are also other commercially available robotic platforms for head and neck surgery such as the Senhance surgical robotic system (TransEnterix, Morrisville, NC), the Single Port Orifice Robotic Technology (SPORT) surgical system (Titan Medical Inc., Toronto, $\mathrm{ON}$ ), and the Versius robotic system (Cambridge Medical Robotics, CMR Surgical, Cambridge, UK). With respect to the head and neck area, a clinical study in which these robotic systems have been used has not yet been published.

\section{Robotic Surgery in Otorhinolaryngology}

Today, surgical robots are occupying a substantial part of otolaryngological surgeries with ongoing development regarding standardization and refinement of procedure steps, patient positioning, and equipment. Robotic surgery in otorhinolaryngology was first performed in a porcine animal model by Haus et al. ${ }^{[25]}$ using the da Vinci surgical system in 2003. In this study, four different types of neck surgeries were successfully applied on both sides of the neck of four animals, and the authors concluded that robotically enhanced endoscopic surgery in the neck was feasible with several compelling advantages over conventional endoscopic neck surgery. Subsequently, after several otolaryngological cadaveric and animal studies conducted with robotic systems, ${ }^{[26-29]}$ McLeod and Melder ${ }^{[30]}$ reported the first utilization of the robot in a human patient. The authors performed a da Vinci-assisted excision of a vallecular cyst in a 46-year-old female patient without any complication. In 2009, the FDA approved the da Vinci system to perform TORS ${ }^{[31]}$, and thenceforward, the manufacturer of robotic surgical systems has been extensively working on the development of more feasible and more sophisticated systems. In a review reported by Weinstein et al. ${ }^{[32]}$ addressing the safety, efficacy, learning process, functional outcomes, oncological outcomes, and cost of TORS, the approach showed several benefits on lowering blood loss, shortening hospital stay, and fewer postoperative complications when compared with open approaches. On the other hand, the disadvantages of robotic surgery are the long initial setup and operative time, and limitations caused by bulky instruments.

Currently, robotic surgery has evolved and is being applied to the surgical management of various otolaryngological diseases. Herein, we provide an overview the current applications of robotic systems in otolaryngology-head and neck surgery.

\section{Oropharynx}

Surgical procedures for oropharyngeal lesions are challenging for surgeons since the oropharynx is a narrow anatomical area with difficult access. In the oropharynx, traditional open surgeries for malignant tumors have been recognized as the gold standard for achieving complete removal of tumors with safe surgical margins. However, open approaches may require mandibulotomy to attain this goal and can cause significant postoperative morbidity, and increase the risk of perioperative complications, long healing periods, and extended hospital stay. Although better visualization of the oropharynx has been achieved with the inclusion of endoscopic systems, limited access and maneuver capacity during surgery stand as a constant problem compelling surgeons to use open approaches. Both TORS and transoral laser microsurgery (TLM) enable good surgical exposure and facilitate total removal of oropharyngeal lesions; thereby, they have become an attractive alternative to open approaches worldwide. Furthermore, with the rapidly evolving technology, TORS has also shown some 
advantages over TLM, such as enabling total resection, providing better access, visualization, and hemostasis control. ${ }^{[3,34]}$

In favor of its unique properties, TORS has rapidly emerged as a treatment alternative for $\mathrm{T} 1$ and $\mathrm{T} 2$ oropharyngeal cancers. ${ }^{[2]}$ Several retrospective studies have been published comparing TORS with other well-established treatment modalities such as definitive radiotherapy (RT) with/without chemotherapy (CT) and conventional open surgery. While lacking the strong evidence of prospective randomized clinical trials, these studies reported comparable oncological and functional outcomes between TORS and other treatment modalities in patients with oropharynx carcinoma. ${ }^{[35-38]}$ However, a Cochrane review highlighted that there was no clear evidence of a change in the treatment of oropharynx carcinoma even though the role of TORS and TLM for the treatment of small-volume oropharynx carcinomas was increasing ${ }^{[39]}$. Of note, despite the increasing number of TORS as a primary treatment of oropharynx carcinoma, adjuvant RT with or without CT is generally applied to the patients after TORS. In the study of Bates et al. ${ }^{\left[{ }^{[0]}\right]}$ including 2514 oropharynx squamous cell carcinoma patients treated with TORS, adjuvant RT and adjuvant CT were used in $75.8 \%$ and $37.5 \%$ of patients, respectively.

In the USA, TORS with concurrent neck dissection is becoming a viable treatment modality for early stage oropharynx squamous cell carcinoma, despite a scarcity of evidence from randomized controlled trials (RCTs). ${ }^{[41]} \mathrm{In}$ 2019, the Eastern Cooperative Oncology Group (ECOG) reported the results of a comparison between primary $\mathrm{RT}$ (with or without CT) and TORS with neck dissection (with or without adjuvant therapy) with regard to quality of life (QOL) in patients with oropharynx carcinoma (ORATOR). ${ }^{[42]}$ As the first randomized study, the authors demonstrated clinically-similar QOL outcomes for both modalities and concluded that a shared decision should be reached after informing patients about both treatment options. Currently, the ECOG 3311 trial, which explored reduced doses of postoperative RT in patients with HPV-associated oropharynx squamous cell carcinoma who underwent primary transoral surgical management (TLM or TORS), met its primary endpoint of 2-year progression-free survival (PFS) ${ }^{[43]}$ The trial had four arms. Arm A consisted of patients with low-risk disease-those with clear margins, 0-1 nodes, and no extranodal extension (ENE), didn't receive any adjuvant therapy. Patients with clear/close margins, 2-4
+ nodes, or ENE $\leq 1 \mathrm{~mm}$ (intermediate-risk) randomized to Arm B and Arm C and received adjuvant RT without CT at lower doses than standard regimen (50 Gy for Arm B and 60 Gy for Arm C). Arm D consisted of high-risk patients for recurrence who had positive margin with any $\mathrm{T}$ stage, $>4+$ nodes or $>1 \mathrm{~mm}$ ENE, received adjuvant 60-66 Gy RT plus weekly $40 \mathrm{mg} / \mathrm{m} 2$ cisplatin. The results of the trial demonstrated that reduced-dose postoperative RT without $\mathrm{C}$ appeared sufficient for patients with intermediate-risk disease. Also, 2-year PFS was favorable for low-risk disease without postoperative therapy (observation alone). The results of the trial summarized in Figure 1.

Carcinoma of unknown primary accounts for about $2-3 \%$ of newly diagnosed head and neck cancers, which has been gradually increasing due to the rise of HPV-associated tumors. ${ }^{[4]}$ Since HPV-associated tumors mostly involve the tonsils and the base of the tongue, TORS has recently been used for identifying the primary tumor site. In a study investigating the primary tumor site of $64 \mathrm{pa}-$ tients with carcinoma of unknown primary utilizing operative laryngoscopy and TORS, an $80 \%$ identification rate of the primary tumor was reported with these two techniques. ${ }^{[45]}$ In this study, 14 primary tumors (22\%) were discovered using operative laryngoscopy alone, however, the rest of the 50 patients underwent TORS lingual tonsillectomy with our without palatine tonsillectomy with the additional identification of primary tumor in 37 patients. In a recent meta-analysis, Meccariello et al. ${ }^{[46]}$ demonstrated comparable efficacy between TORS and TLM for the identification of primary tumor sites, especially in the base of tongue where the technical advantages of robotic systems becomes prominent.

\section{Larynx and Hypopharynx}

Although the vast majority of studies on TORS were conducted for the management of oropharyngeal lesions, technological advancements have led to the robotic systems being useful for the treatment of laryngeal lesions. However, the current architecture of the robotic systems is mostly applied to resection of supraglottic tumors due to the limited access of the glottis and subglottis. TORS seems to be a safe, useful, and effective approach for supraglottic laryngectomy. ${ }^{[4]}$ In the literature, there is a paucity of studies comparing TORS with other alternative treatment methods, particularly for supraglottic lesions. Slama et al. ${ }^{[4]}$ compared the outcomes of TORS and open surgery in patients with early-stage supraglottic larynx 


\section{3 patients}

cT1-2, stage III/IV AJCC7

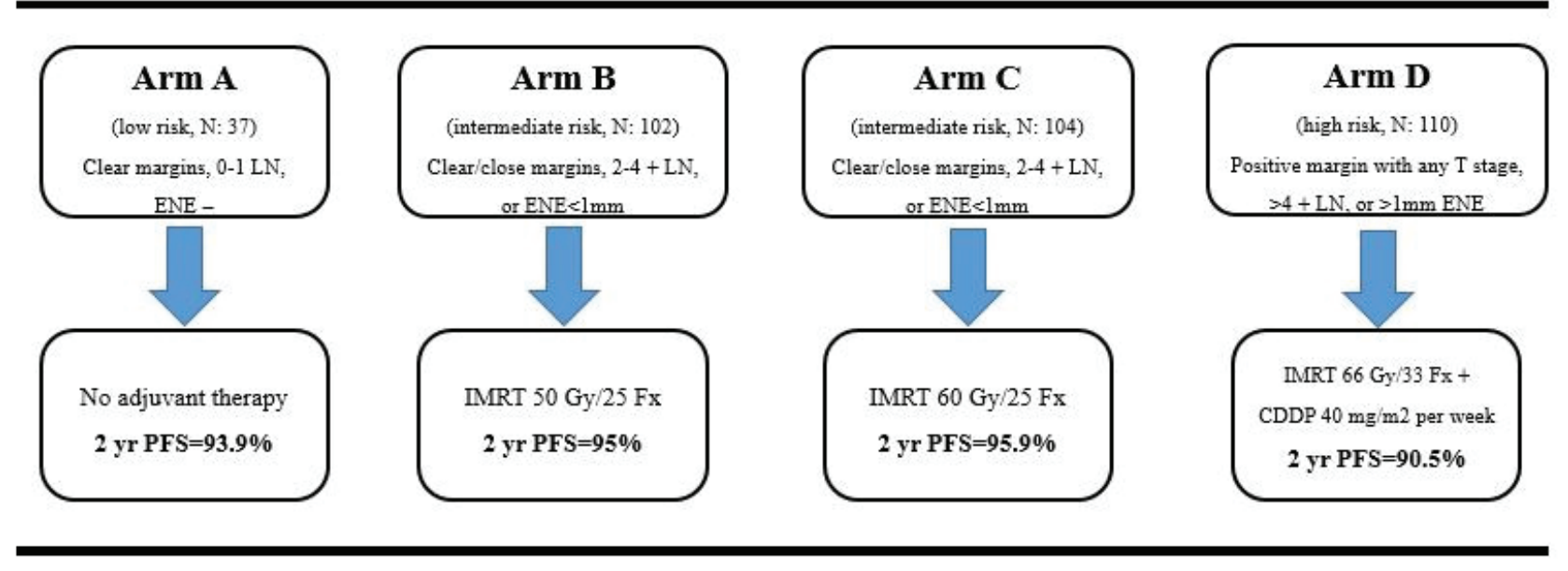

Figure 1. The results of the Eastern Cooperative Oncology Group (ECOG) 3311 clinical trial. The trial included 353 oropharynx carcinoma patients with CT1-2 stage III/IV AJCC7 without matted neck nodes who underwent transoral resection of the tumor by 82 credentialed surgeons. (CDDP: cisplatin, ENE: extranodal extension, Fx: fractions, IMRT: intensity-modulated radiation, LN: lymph node, $\mathrm{N}$ : number of patients, PFS: progression-free survival, yr: year.

cancer and determined that TORS significantly reduces intraoperative blood loss, pain, and psychosocial distress, and increases swallowing function postoperatively. Asik et al. ${ }^{[4]}$ performed a meta-analysis in which the effectiveness of TORS and TLM were analyzed, and compared their respective oncologic outcomes of patients with supraglottic larynx cancers. The authors found comparable and acceptable oncological outcomes between the two procedures. However, there is no study comparing TORS with curative RT with or without CT for the treatment of supraglottic larynx cancers.

There is some evidence to suggest that TORS may be a safe, and reliable approach for performing total laryngectomy (TL). ${ }^{[50,51]}$ Smith et al. ${ }^{[50]}$ suggested TORS as an excellent method for salvage TL in patients with limited glottic disease. In a recent study including seven recurrent laryngeal cancers without neck metastasis, the 36-month long-term survival rate after TORS-assisted TL was $86 \%$ while the morbidity was low, and patient swallowing function was satisfactory. ${ }^{[52]}$

Although the glottic level is an off-label use of the da Vinci system, TORS was reported as feasible and effective for early-stage glottic carcinomas. ${ }^{[53,54]}$ In 2019, Kayhan et al. ${ }^{[5]}$ reported oncological outcomes of 48 patients with early-stage glottic carcinoma (Tis, T1, T2) who underwent cordectomy using the da Vinci system. The mean follow-up time was $65.6 \pm 16.6$ months, and TORS had similar results to those of TLM and RT in terms of laryngeal preservation, recurrence, and survival rates. However, it seems that more focus on innovative developments oriented to the glottic area is essential prior to widening the utilization of TORS for early-stage glottic carcinomas.

Over the past decades, total pharyngolaryngectomy has constituted the main surgical treatment modality for advanced-stage hypopharyngeal carcinoma with significant morbidity. Although organ preservation protocols with more effective CT and conformal RT techniques provide more functional treatment outcomes, the prognosis has remained stable. ${ }^{[56]}$ Recently, transoral resection of hypopharyngeal tumors utilizing TORS has been deployed for the treatment of hypopharyngeal carcinomas with promising results. Hassid et al. ${ }^{[57]}$ reported the results of 22 hypopharyngectomies performed via TORS with curative intent. They demonstrated that TORS hypopharyngecto- 
my is a safe procedure with reduced postoperative morbidity compared to the open approach. Also, the authors found shorter hospital stays and faster recovery of safe swallowing with TORS hypopharyngectomy. In a systematic review and meta-analysis, De Virgilio et al. ${ }^{[5]}$ investigated the role of minimally invasive surgical approaches such as TLM and TORS for the treatment of hypopharyngeal carcinoma. At 36 months follow-up, the cumulative survival rate for TORS and TLM subgroups was $85.5 \%$ and $58.5 \%$, respectively. However, the authors noted that TLM studies included a lower proportion of patients with earlier stage disease than TORS studies.

\section{Thyroid, parathyroid and neck}

The main advantage of robotic thyroidectomy is avoiding the anterior neck scar following conventional thyroid surgery, in other words "scar-less" neck surgery. Anterior neck scars may cause psychological distress and decreased QOL. ${ }^{[59]}$ Robotic thyroidectomy has been described through multiple routes including transaxillary, bilateral axillo-breast, facelift, and transoral approaches. In 2009, Kang et al. ${ }^{[60]}$ reported the first case series (100 patients with papillary thyroid cancer) of robotic thyroidectomy using a gasless transaxillary approach Thereafter, several case series demonstrated that the surgical and oncological outcomes of both conventional and robotic thyroidectomy were similar. ${ }^{[2,61]}$ However, in a review of the National Cancer Database of the United States, robotic thyroid surgery was found to be associated with increased risk of positive margins. ${ }^{[62]}$

Following the first robotic thyroidectomy, robotic parathyroidectomy was first reported in London, UK, in 2011. ${ }^{[63]}$ In this study, the authors demonstrated the feasibility of a robotic approach for performing targeted parathyroidectomy in 11 patients with primary hyperparathyroidism. Tolley et al. ${ }^{[64]}$ reported that robotic parathyroidectomy provides superior early cosmesis with equivalent global health improvement compared to focused lateral parathyroidectomy. Although robotic parathyroidectomy provides a scar-less neck, the high cost and the necessity of a learning curve may hamper its adoption worldwide.

Robotic surgery was also reported for performing neck dissections. In 2010, Kang et al. ${ }^{[65]}$ reported their case series of 33 patients with thyroid cancer and lateral neck LN metastases who underwent robot-assisted thyroidectomy and robotic modified radical neck dissection using a transaxillary approach. The authors found robot-assisted modified radical neck dissection to be technically feasible and safe with excellent cosmetic results. In 2012, Kim et al. ${ }^{[66]}$ introduced a combined transaxillary- retroauricular approach to facilitate dissection levels I, IIb, and Va. A recent meta-analysis reported that robotic neck dissection provides comparable results with a conventional approach in terms of nodal yields, recurrence and complication rates but it requires significantly longer operative times than conventional neck dissection. ${ }^{[67]}$

Robotic surgical removal of the submandibular gland via a transoral or trans-hairline approach was reported to be feasible and safe. ${ }^{[68,69]}$ Robotic systems have been used for submandibular sialolith removal since 2011. ${ }^{[70]}$ Tampio et al. ${ }^{[71]}$ described the advantages of robot-assisted sialolithotomy with sialoendoscopy as a high success rate of sialolith removal, lower risk of permanent lingual nerve damage, and temporary tongue paresthesia.

\section{Skull Base}

In the past several years, minimally invasive approaches have become more popular than open conventional surgeries for the management of neoplastic and non-neoplastic lesions of the anterior and central skull base. Minimally invasive surgical techniques involve smaller incisions and more preservation of healthy tissues that make them more appealing approaches for many skull base pathologies. ${ }^{[72]}$ The transnasal endoscopic approach is the most preferred treatment modality of minimally invasive surgery for skull base pathologies. However, robotic surgery with or without a transnasal endoscopic approach has recently been studied for feasibility in the management of skull base pathologies.

In 2007, Hanna et al. ${ }^{[73}$ described a novel robotic endoscopic surgery using the da Vinci surgical system in human cadavers that allows adequate endoscopic access of the anterior and central skull base. In this study, the authors reported that robotic-assisted endoscopic surgery provided 3-dimensional, 2-handed, tremor-free endoscopic dissection, and precise closure of dural defects during skull base surgery. In another study by the same group reported in $2009^{[74]}$, they described a novel approach using transantral robotic access that allows endoscopic access for resection of tumors involving the pituitary fossa. The authors reported that transantral robotic surgery ensures stereoscopic endoscopic access to the pituitary gland and the optic chiasm with bimanual endoscopic manipulation of the pituitary gland and surrounding structures. Furthermore, O'Malley and Weinstein ${ }^{[75]}$ performed robotic anterior and midline 
skull base surgery procedures focusing on approaches to the nasopharynx, clivus, sphenoid, pituitary sellar, and suprasellar regions on one cadaver and one live mongrel dog using the da Vinci surgical robot. The authors stated the potential application of robotic surgeries for minimally invasive access and surgery of anterior and midline skull base surgeries. To date, several reports have been published in the literature regarding the utilization of robots in the surgical treatment of skull base surgery that mostly were performed using a hybrid approach combining an endonasal- transantral approach with a transoral-transcervical approach. ${ }^{[2]}$ Although robotic surgeries for the skull base are under development, many advantages of these systems have been reported, including stereoscopic endoscopic access to the anterior skull base and pituitary fossa, which allows tremor-free and 2-handed endoscopic manipulation and reconstruction. ${ }^{[72,76]}$ Nevertheless, it is still early to adopt robotic-assisted skull base surgery due to several challenges. Limited access, the absence of a drill, no integrated image navigation, and the lack of haptic feedback still stand as drawbacks of robotic systems in skull base surgery. However, cadaveric studies have shown that contemporary robotic systems with SP design may allow better access to the skull base. ${ }^{[77]}$ Future developments regarding the flexibility of the robotic systems and refinement of endonasal robotic instrumentation seem vital for increasing the role of robotic surgery in the management of skull base pathologies.

\section{Obstructive sleep apnea and Other Sites}

Obstructive sleep apnea surgery is one of the surgical fields in which TORS has been utilized successfully. In 2010, TORS for OSA was first performed by Vicini et al. ${ }^{[78]}$ in OSA patients with tongue base hypertrophy and the authors concluded that TORS was effective and functional in terms of pain, swallowing, and QOL. Since then, an increasing body of evidence supports the efficacy and safety of TORS in the treatment of moderate-to-severe OSA. In the review of Garas et al. ${ }^{[79]}$, TORS was shown to be an effective treatment modality in more than $75 \%$ of non-obese OSA patients and more than $50 \%$ of non-morbidly obese OSA patients. Although TORS seems to be an advantageous surgical modality given its superior visualization and ergonomics, the favorable results regarding the efficacy of TORS in OSA surgery should be supported by RCTs.

Ozer and Waltonen ${ }^{[80]}$ first performed surgery with TORS for the nasopharynx on a human cadaver. In this preclinical investigation, the authors demonstrated that
TORS was a feasible surgical technique for nasopharyngeal lesions. As a minimally invasive approach for the surgical treatment of recurrent nasopharynx carcinoma, robotic nasopharynx surgeries were initially hampered due to the necessity of soft palate splitting in an irradiated field and lack of bone drills. However, through advancements of robotic technologies, the palate-sparing approach was shown to be feasible with Flex ${ }^{\circledR}$ and Vinci SP robotic systems in pre-clinical studies. ${ }^{[81,82]}$ Further clinical studies investigating the feasibility of robotic nasopharyngectomy without palate-splitting may clear the way for robotic surgeries in the treatment of nasopharynx carcinomas.

The parapharyngeal space (PPS) has a deep location in the neck with highly complex anatomy. Therefore, numerous surgical approaches have been introduced for the removal of PPS tumors. ${ }^{[83]}$ TORS was demonstrated to facilitate access to the PPS by excluding the morbidity of cervical incision and mandibulotomy. ${ }^{[84]}$ Chan et al. ${ }^{[85]}$ emphasized that TORS is useful for the removal of PPS tumors with minimal surgical morbidity. However, caution should be used especially for pleomorphic adenomas to avoid capsule violations. Chu et al. ${ }^{[86]}$ reported the retrospective analysis of 17 patients with a PPS tumor treated by TORS or combined TORS and transcervical approach. The authors concluded that for well-capsulated PPS tumors, TORS is a safe and effective approach, however, for large and/or invasive tumors the combined robotic-assisted transoral/transcervical approach may provide better oncological outcomes.

\section{The future of robotic surgery in otolaryngology}

Today, robotic surgery has advanced far beyond its introduction, and in the foreseeable future its capabilities will likely be better than today. Robotic systems seem to have a great capacity for facilitating surgery in the head and neck region, and the clinical utilization of robots in otolaryngology-head neck practice is expanding. However, currently the most prominent shortcoming of robotic systems is the high cost, similar to that of other highly-sophisticated technological devices, but this problem may soon be solved with the expiration of patents and increased market competition. ${ }^{[87,88]}$

Medical fields such as otorhinolaryngology that deal with anatomical regions with narrow spaces and limited corridors for access pose a substantial limitation for robotic systems due to the bulky arms of the robots. Indeed, more flexibility and a decrease in instrumentation size are 
required to facilitate the movements of the equipment. The Flex ${ }^{\circledR}$ robotic system, individually designed for head and neck surgery ${ }^{[20]}$, promises more flexibility through the existence of more flexible subunits at a lower cost. ${ }^{\left[{ }^{[9]}\right.} \mathrm{Al}-$ though the first generations of da Vinci robots posed limitations in otolaryngological procedures due to the rigid and bulky arms, the majority of these limitations have been overcome with the launch of the da Vinci single-port (SP) system. Hence, the SP system promises a better application for the lateral oropharyngeal, tongue base, hypopharynx, nasopharynx, and skull base than earlier systems. ${ }^{[2]}$ In the future, robotic systems with more flexibility and smaller size should increase their wider adoption in otolaryngology and head and neck surgery.

Several technological features to improve surgical precision and safety such as augmented reality for image guidance, haptic feedback, and microendoscopic systems have been under development for integration into the robotic surgical systems, although their efficacy and feasibility have not yet been confirmed by clinical studies. ${ }^{[0,91]}$ Augmented reality for image guidance involves mental 3D pictures created from preoperative radiological images that assist in resecting tumors with safe margins while preserving critical anatomical structures. The early application of this system was performed by a separate image guidance system (BrainLAB AG, Feldkirchen, Germany), ${ }^{[92]}$ whereas augmented reality for an image guidance system integrated into the latest da Vinci system is called the "TilePro" function. ${ }^{[2]}$

One of the main limitations of the current robotic systems is the lack of haptic feedback. Haptics general touch feedback involves kinesthetic (force) and cutaneous (tactile) feedback. ${ }^{[93]}$ Although some of the newer robotic systems include haptic feedback (FLEX ${ }^{\circledR}$, Senhance, Versius), the technology is still in its infancy. Friedrich et al. ${ }^{\text {[1] }}$ compared the human hand with the da Vinci system, the Flex ${ }^{\circledR}$ system, and standard rigid instruments (23 cm laryngoscopic grasper, Karl Storz) in an experimental study to determine the differences in terms of haptic feedback while performing a variety of surgical tasks. In this study, standard rigid instruments demonstrated similar performance to that of human hands, whereas the robotic systems showed lower achievement. Although the Flex ${ }^{\circledR}$ system had a better performance than the da Vinci robot, the authors showed the necessity of further studies conducted with more complex tasks that are closer to clinical procedures.

As is well-defined in the literature, obtaining clear sur- gical margins has paramount importance in oncological surgery. ${ }^{[94]}$ TORS has several disadvantages with regard to tumor margins such as difficulty with specimen orientation and the absence of haptic feedback. In the current approach, surgeons utilize frozen sections and inking of specimens during TORS to overcome these shortcomings. However, various studies have investigated possible alternatives for examining resection margins during TORS. Patsias et al. ${ }^{[5]}$ introduced a miniaturized high-resolution fiberoptic microendoscope integrated with the da Vinci robotic system that provides real-time histological assessment. They demonstrated that the system facilitates a safe and technically feasible approach in the oropharynx. Similarly, Gorpas et al. ${ }^{[96]}$ introduced time-resolved fluorescence spectroscopy integrated with the da Vinci robot that highlights tumor tissue with its distinct autofluorescence signature during TORS in real-time. Further developments in optical technology and delivery systems may help to better assess the surgical margins during TORS tumor surgery.

Finally, autonomous surgical robotic systems for performing high-precision surgeries such as mastoidectomy, ${ }^{[97]}$ cochlear implantation, ${ }^{[98]}$ and the resection of vestibular schwannoma ${ }^{[99]}$ have recently been under development. Autonomous surgical robotic systems require incorporation of preoperative radiological data with an accurate hardware and guidance system to benefit from the precision of surgical navigation and robotic surgery. Although the initial steps of autonomous robotic surgeries are just being explored, "surgeon-less surgery" with autonomous robots may become a reality in the future.

\section{Conclusion}

Robotic technologies have become a substantial part of human life with ongoing progress, and medical devices have also gained their share with increasing popularization. Robotic surgery is one of the most prominent innovative developments seen in the surgical field during the last two decades. In the field of otolaryngology-head and neck surgery, robotic systems may be beneficial allowing better access to and visualization of multiple narrow anatomical regions. Accordingly, TORS has become a viable alternative for the treatment of oropharyngeal diseases. However, better flexibility, reduced size, lower cost, and the integration of additional technologies such as augmented reality and haptic feedback may further expand the utilization of robotic systems in Otolaryngology-Head and Neck Surgery. 


\section{References}

1. Poon H, Li C, Gao W, Ren H, Lim CM. Evolution of robotic systems for transoral head and neck surgery. Oral Oncol 2018;87:82-8.

2. Tamaki A, Rocco JW, Ozer E. The future of robotic surgery in otolaryngology - head and neck surgery. Oral Oncol 2020 Feb; doi: 10.1016/j. oraloncology.2019.104510. [Epub ahead of print]

3. de Almeida JR, Li R, Magnuson JS, et al. Oncologic Outcomes After Transoral Robotic Surgery: a Multi-institutional Study. JAMA Otolaryngol Head Neck Surg 2015;141:1043-51.

4. Park YM, Lee WJ, Lee JG, et al. Transoral robotic surgery (TORS) in laryngeal and hypopharyngeal cancer. J Laparoendosc Adv Surg Tech A 2009;19:361-8.

5. Weinstein GS, O'Malley BW Jr, Snyder W, Hockstein NG. Transoral robotic surgery: supraglottic partial laryngectomy. Ann Otol Rhinol Laryngol 2007;116:19-23.

6. Ciabatti PG, Burali G, D’Ascanio L. Transoral robotic surgery for large mixed laryngocoele. J Laryngol Otol 2013;127:435-7.

7. Ghanem TA. Transoral robotic-assisted microvascular reconstruction of the oropharynx. Laryngoscope 2011;121:580-2.

8. Ruhle BC, Ferguson Bryan A, Grogan RH. Robot-Assisted Endocrine Surgery: Indications and Drawbacks. J Laparoendosc Adv Surg Tech A 2019;29:129-35.

9. Rangarajan S, Hachem RA, Ozer E, Beer-Furlan A, Prevedello D, Carrau RL. Robotics in Sinus and Skull Base Surgery. Otolaryngol Clin North Am 2017;50:633-41.

10. Cammaroto G, Stringa LM, Zhang H, et al. Alternative Applications of Trans-Oral Robotic Surgery (TORS): a Systematic Review. J Clin Med 2020;9:201.

11. Nakayama M, Holsinger FC, Chevalier D, Orosco RK. The dawn of robotic surgery in otolaryngology-head and neck surgery. Jpn J Clin Oncol 2019;49:404-11

12. Mohammad S. Robotic surgery. J Oral Biol Craniofac Res 2013;3:2.

13. Camarillo DB, Krummel TM, Salisbury JK Jr. Robotic technology in surgery: past, present, and future. Am J Surg 2004;188:2-15.

14. Falcone T, Goldberg J, Garcia-Ruiz A, Margossian H, Stevens L. Full robotic assistance for laparoscopic tubal anastomosis: a case report. J Laparoendosc Adv Surg Tech A 1999;9:107-13.

15. Marescaux J, Leroy J, Gagner M, et al. Transatlantic robot-assisted telesurgery. Nature 2001;413:379-80.

16. Moran ME. The da Vinci robot. J Endourol 2006;20:986-90.

17. U.S. Food and Drug Administration. 510(k) Premarket Notification Number: K990144; 2000.

18. U.S. Food and Drug Administration. 510(k) Premarket Notification Number: K182371; 2019.

19. Rivera-Serrano CM, Johnson P, Zubiate B, et al. A transoral highly flexible robot: Novel technology and application. Laryngoscope 2012;122:1067-71.

20. Remacle M, M N Prasad V, Lawson G, Plisson L, Bachy V, Van der Vorst S. Transoral robotic surgery (TORS) with the Medrobotics Flex ${ }^{\mathrm{TM}}$ System: first surgical application on humans. Eur Arch Otorhinolaryngol 2015;272:1451-5.

21. Mandapathil M, Duvvuri U, Güldner C, Teymoortash A, Lawson G, Wer- ner JA. Transoral surgery for oropharyngeal tumors using the Medrobot-

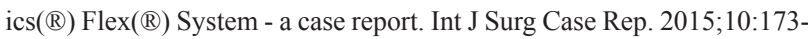
5 .

22. Mattheis S, Lang S. [A new flexible endoscopy-system for the transoral resection of head and neck tumors]. [Article in German] Laryngorhinootologie 2015;94:25-8.

23. U.S. Food and Drug Administration. 510(k) Premarket Notification Number: K150776; 2015.

24. Hussain T, Lang S, Haßkamp P, Holtmann L, Höing B, Mattheis S. The Flex robotic system compared to transoral laser microsurgery for the resection of supraglottic carcinomas: first results and preliminary oncologic outcomes. Eur Arch Otorhinolaryngol 2020;277:917-24.

25. Haus BM, Kambham N, Le D, Moll FM, Gourin C, Terris DJ. Surgical robotic applications in otolaryngology. Laryngoscope 2003;113:113944.

26. Weinstein GS, O'malley BW Jr, Hockstein NG. Transoral robotic surgery: supraglottic laryngectomy in a canine model. Laryngoscope 2005;115:1315-9.

27. Hockstein NG, Nolan JP, O'malley BW Jr, Woo YJ. Robotic microlaryngeal surgery: a technical feasibility study using the daVinci surgical robot and an airway mannequin. Laryngoscope 2005;115:780-5.

28. Hockstein NG, Nolan JP, O’Malley BW Jr, Woo YJ. Robot-assisted pharyngeal and laryngeal microsurgery: results of robotic cadaver dissections. Laryngoscope 2005;115:1003-8.

29. Hockstein NG, Weinstein GS, O'malley BW Jr. Maintenance of hemostasis in transoral robotic surgery. ORL J Otorhinolaryngol Relat Spec 2005;67:220-4.

30. McLeod IK, Melder PC. Da Vinci robot-assisted excision of a vallecular cyst: a case report. Ear Nose Throat J 2005;84:170-2.

31. U.S. Food and Drug Administration. 510(k) Premarket Notification Number: K090993; 2009

32. Weinstein GS, O'Malley BW Jr, Desai SC, Quon H. Transoral robotic surgery: does the ends justify the means? Curr Opin Otolaryngol Head Neck Surg 2009;17:126-31.

33. Lörincz BB, Jowett N, Knecht R. Decision management in transoral robotic surgery: Indications, individual patient selection, and role in the multidisciplinary treatment for head and neck cancer from a European perspective. Head Neck 2016;38:2190-6.

34. Moorthy K, Munz Y, Dosis A, et al. Dexterity enhancement with robotic surgery. Surg Endosc 2004;18:790-5.

35. Gangwani K, Shetty L, Seshagiri R, Kulkarni D. Comparison of TORS with Conventional Surgery for Oropharyngeal Carcinomas in T1-T4 Lesions. Ann Maxillofac Surg 2019;9:387-392.

36. de Almeida JR, Byrd JK, Wu R, et al. A systematic review of transoral robotic surgery and radiotherapy for early oropharynx cancer: a systematic review. Laryngoscope 2014;124:2096-102.

37. Chen AM, Daly ME, Luu Q, Donald PJ, Farwell DG. Comparison of functional outcomes and quality of life between transoral surgery and definitive chemoradiotherapy for oropharyngeal cancer. Head Neck 2015;37:381-5.

38. Moore EJ, Olsen SM, Laborde RR, et al. Long-term functional and on- 
cologic results of transoral robotic surgery for oropharyngeal squamous cell carcinoma. Mayo Clin Proc 2012;87:219-25.

39. Howard J, Masterson L, Dwivedi RC, et al. Minimally invasive surgery versus radiotherapy/chemoradiotherapy for small-volume primary oropharyngeal carcinoma. Cochrane Database Syst Rev 2016;12:CD010963.

40. Bates JE, Hitchcock KE, Mendenhall WM, Dziegielewski PT, Amdur RJ. Comparing national practice versus standard guidelines for the use of adjuvant treatment following robotic surgery for oropharyngeal squamous cell carcinoma. Head Neck 2020;42:2602-6.

41. Cracchiolo JR, Baxi SS, Morris LG, et al. Increase in primary surgical treatment of T1 and T2 oropharyngeal squamous cell carcinoma and rates of adverse pathologic features: National Cancer Data Base. Cancer 2016;122:1523-32.

42. Nichols AC, Theurer J, Prisman E, et al. Radiotherapy versus transoral robotic surgery and neck dissection for oropharyngeal squamous cell carcinoma (ORATOR): an open-label, phase 2, randomised trial. Lancet Oncol. 2019;20:1349-59.

43. Ferris RL, Flamand Y, Weinstein GS, et al. Transoral robotic surgical resection followed by randomization to low- or standard-dose IMRT in resectable p16+ locally advanced oropharynx cancer: A trial of the ECOG-ACRIN Cancer Research Group (E3311). J Clin Oncol 38: 2020 (suppl; abstr 6500).

44. Mehta V, Johnson P, Tassler A, et al. A new paradigm for the diagnosis and management of unknown primary tumors of the head and neck: a role for transoral robotic surgery. Laryngoscope 2013;123:146-51.

45. Geltzeiler M, Doerfler S, Turner M, et al. Transoral robotic surgery for management of cervical unknown primary squamous cell carcinoma: Updates on efficacy, surgical technique and margin status. Oral Oncol 2017;66:9-13.

46. Meccariello G, Cammaroto G, Ofo E, et al. The emerging role of transoral robotic surgery for the detection of the primary tumour site in patients with head-neck unknown primary cancers: A meta-analysis. Auris Nasus Larynx. 2019;46:663-71.

47. Lechien JR, Fakhry N, Saussez S, et al. Surgical, clinical and functional outcomes of transoral robotic surgery for supraglottic laryngeal cancers: A systematic review. Oral Oncol 2020 Jun; doi: 10.1016/j.oraloncology.2020.104848. [Epub ahead of print].

48. Slama K, Slouka D, Slipka J, Fischer S. Short-term postoperative distress associated with open vs. transoral robotic surgery (TORS) in patients with T1-T2 carcinomas of the tongue base and supraglottis. Biomed Pap Med Fac Univ Palacky Olomouc Czech Repub 2016;160:423-8.

49. Asik MB, Satar B, Serdar M. Meta-analytic comparison of robotic and transoral laser surgical procedures in supraglottic carcinoma. J Laryngol Otol 2019;133:404-12.

50. Smith RV, Schiff BA, Sarta C, Hans S, Brasnu D. Transoral robotic total laryngectomy. Laryngoscope 2013;123:678-82.

51. Lawson G, Mendelsohn A, Fakhoury R, et al. Transoral Robotic Surgery Total Laryngectomy. ORL J Otorhinolaryngol Relat Spec 2018;80:171-7.

52. Wang CC, Lin WJ, De Virgilio A, Liu SA, Chen SH, Liao JS. Long-term outcomes of trans-oral robotic surgery-assisted total laryngectomy for recurrent laryngeal cancers. Jpn J Clin Oncol 2020;50:653-60.

53. Kayhan FT, Kaya KH, Sayin I. Transoral robotic cordectomy for early glottic carcinoma. Ann Otol Rhinol Laryngol 2012;121:497-502.

54. Lallemant B, Chambon G, Garrel R, et al. Transoral robotic surgery for the treatment of T1-T2 carcinoma of the larynx: preliminary study. Laryngoscope. 2013;123:2485-90.

55. Kayhan FT, Koc AK, Erdim I. Oncological outcomes of early glottic carcinoma treated with transoral robotic surgery. Auris Nasus Larynx 2019;46:285-93.

56. Persky MS, Daly JF. Combined therapy vs curative radiation in the treatment of pyriform sinus carcinoma. Otolaryngol Head Neck Surg 1981;89:87-91.

57. Hassid S, Van der Vorst S, Delahaut G, Ambroise J, Lawson G. Transoral robotic surgery hypopharyngectomy (TORSH): feasibility and outcomes. Eur Arch Otorhinolaryngol 2020 May; doi: 10.1007/s00405-02005984-y. [Epub ahead of print].

58. De Virgilio A, Iocca O, Malvezzi L, et al. The Emerging Role of Robotic Surgery among Minimally Invasive Surgical Approaches in the Treatment of Hypopharyngeal Carcinoma: Systematic Review and Meta-Analysis. J Clin Med 2019;8:256.

59. Choi Y, Lee JH, Kim YH, et al. Impact of postthyroidectomy scar on the quality of life of thyroid cancer patients. Ann Dermatol 2014;26:693-9.

60. Kang SW, Jeong JJ, Yun JS, et al. Robot-assisted endoscopic surgery for thyroid cancer: experience with the first 100 patients. Surg Endosc 2009;23:2399-406.

61. Kim K, Lee S, Bae JS, Kim JS. Comparison of long-term surgical outcome between transaxillary endoscopic and conventional open thyroidectomy in patients with differentiated thyroid carcinoma: a propensity score matching study. Surg Endosc 2020 Jun; doi: 10.1007/s00464-02007721-2. [Epub ahead of print].

62. Jacobs D, Torabi SJ, Gibson C, Rahmati R, Mehra S, Judson BL. Assessing National Utilization Trends and Outcomes of Robotic and Endoscopic Thyroidectomy in the United States. Otolaryngol Head Neck Surg 2020 Jun; doi: 10.1177/0194599820927699. [Epub ahead of print].

63. Tolley N, Arora A, Palazzo F, et al. Robotic-assisted parathyroidectomy: a feasibility study. Otolaryngol Head Neck Surg 2011;144:859-66.

64. Tolley N, Garas G, Palazzo F, et al. Long-term prospective evaluation comparing robotic parathyroidectomy with minimally invasive open parathyroidectomy for primary hyperparathyroidism. Head Neck 2016;38:300-6.

65. Kang SW, Lee SH, Ryu HR, et al. Initial experience with robot-assisted modified radical neck dissection for the management of thyroid carcinoma with lateral neck node metastasis. Surgery 2010;148:1214-21.

66. Kim WS, Lee HS, Kang SM, et al. Feasibility of robot-assisted neck dissections via a transaxillary and retroauricular ("TARA") approach in head and neck cancer: preliminary results. Ann Surg Oncol 2012;19:1009-17.

67. Sukato DC, Ballard DP, Abramowitz JM, Rosenfeld RM, Mlot S. Robotic versus conventional neck dissection: A systematic review and meta-analysis. Laryngoscope 2019;129:1587-96.

68. Prosser JD, Bush CM, Solares CA, Brown JJ. Trans-oral robotic submandibular gland removal. J Robot Surg 2013;7:87-90.

69. Yang TL. Robotic surgery for submandibular gland resection through a trans-hairline approach: The first human series and comparison with applicable approaches. Head Neck 2018;40:793-800.

70. Walvekar RR, Tyler PD, Tammareddi N, Peters G. Robotic-assisted transoral removal of a submandibular megalith. Laryngoscope 2011;121:5347. 
71. Tampio AJF, Marzouk MF. Robot-assisted sialolithotomy with sialoendoscopy: a review of safety, efficacy and cost. J Robot Surg 2020 May 29; doi: 10.1007/s11701-020-01097-0. [Epub ahead of print].

72. Kupferman ME, Hanna E. Robotic surgery of the skull base. Otolaryngol Clin North Am 2014;47:415-23.

73. Hanna EY, Holsinger C, DeMonte F, Kupferman M. Robotic endoscopic surgery of the skull base: a novel surgical approach. Arch Otolaryngol Head Neck Surg 2007;133:1209-14.

74. Kupferman M, Demonte F, Holsinger FC, Hanna E. Transantral robotic access to the pituitary gland. Otolaryngol Head Neck Surg 2009;141:4135 .

75. O’Malley BW Jr, Weinstein GS. Robotic anterior and midline skull base surgery: preclinical investigations. Int J Radiat Oncol Biol Phys 2007;69:125-8.

76. Kupferman ME, Demonte F, Levine N, Hanna E. Feasibility of a robotic surgical approach to reconstruct the skull base. Skull Base 2011;21:7982.

77. Schuler PJ, Scheithauer M, Rotter N, Veit J, Duvvuri U, Hoffmann TK. A single-port operator-controlled flexible endoscope system for endoscopic skull base surgery. HNO 2015;63:189-94.

78. Vicini C, Dallan I, Canzi P, Frassineti S, La Pietra MG, Montevecchi F. Transoral robotic tongue base resection in obstructive sleep apnoea-hypopnoea syndrome: a preliminary report. ORL J Otorhinolaryngol Relat Spec 2010;72:22-7.

79. Garas G, Kythreotou A, Georgalas C, et al. Is transoral robotic surgery a safe and effective multilevel treatment for obstructive sleep apnoea in obese patients following failure of conventional treatment(s)? Ann Med Surg (Lond) 2017;19:55-61.

80. Ozer E, Waltonen J. Transoral robotic nasopharyngectomy: a novel approach for nasopharyngeal lesions. Laryngoscope 2008;118:1613-6.

81. Richmon JD. Transoral palate-sparing nasopharyngectomy with the Flex® System: preclinical study. Laryngoscope 2015;125:318-22.

82. Tsang RK, Holsinger FC. Transoral endoscopic nasopharyngectomy with a flexible next-generation robotic surgical system. Laryngoscope 2016;126:2257-62.

83. López F, Suárez C, Vander Poorten V, et al. Contemporary management of primary parapharyngeal space tumors. Head Neck 2019;41:522-35.

84. Chu F, Tagliabue M, Giugliano G, Calabrese L, Preda L, Ansarin M. From transmandibular to transoral robotic approach for parapharyngeal space tumors. Am J Otolaryngol 2017;38:375-9.

85. Chan JY, Tsang RK, Eisele DW, Richmon JD. Transoral robotic surgery of the parapharyngeal space: a case series and systematic review. Head Neck 2015;37:293-8.

86. Chu F, De Berardinis R, Tagliabue M, Zorzi S, Bandi F, Ansarin M. The Role of Transoral Robotic Surgery for Parapharyngeal Space: Experience of a Tertiary Center. J Craniofac Surg 2020;31:117-20.

87. Garas G, Cingolani I, Panzarasa P, Darzi A, Athanasiou T. Network analysis of surgical innovation: Measuring value and the virality of diffusion in robotic surgery. PLoS One 2017;12:e0183332.

88. Malik HT, Marti J, Darzi A, Mossialos E. Savings from reducing low-value general surgical interventions. Br J Surg 2018;105:13-25.

89. Garas G, Tolley N. Robotics in otorhinolaryngology - head and neck surgery. Ann R Coll Surg Engl 2018;100:34-41.

90. Chan JYK, Holsinger FC, Liu S, Sorger JM, Azizian M, Tsang RKY. Augmented reality for image guidance in transoral robotic surgery. J Robot Surg 2020;14:579-83.

91. Friedrich DT, Dürselen L, Mayer B, et al. Features of haptic and tactile feedback in TORS-a comparison of available surgical systems. J Robot Surg 2018;12:103-8.

92. Desai SC, Sung CK, Genden EM. Transoral robotic surgery using an image guidance system. Laryngoscope 2008;118:2003-5.

93. Okamura AM. Haptic feedback in robot-assisted minimally invasive surgery. Curr Opin Urol 2009;19:102-7.

94. Haque R, Contreras R, McNicoll MP, Eckberg EC, Petitti DB. Surgical margins and survival after head and neck cancer surgery. BMC Ear Nose Throat Disord 2006;6:2.

95. Patsias A, Giraldez-Rodriguez L, Polydorides AD, et al. Feasibility of transoral robotic-assisted high-resolution microendoscopic imaging of oropharyngeal squamous cell carcinoma. Head Neck 2015;37:99-102.

96. Gorpas D, Phipps J, Bec J, et al. Autofluorescence lifetime augmented reality as a means for real-time robotic surgery guidance in human patients. Sci Rep 2019;9:1187.

97. Yoo MH, Lee HS, Yang CJ, et al. A cadaver study of mastoidectomy using an image-guided human-robot collaborative control system. Laryngoscope Investig Otolaryngol 2017;2:208-14.

98. Caversaccio M, Gavaghan K, Wimmer W, et al. Robotic cochlear implantation: surgical procedure and first clinical experience. Acta Otolaryngol 2017; 137:447-54

99. Dillon NP, Balachandran R, Siebold MA, Webster RJ 3rd, Wanna GB, Labadie RF. Cadaveric Testing of Robot-Assisted Access to the Internal Auditory Canal for Vestibular Schwannoma Removal. Otol Neurotol 2017;38:441-7.

Conflict of Interest: The authors have no conflicts of interest to declare.

Financial Disclosure: The authors declare that this study has received no financial support.

This is an open access article distributed under the terms of the Creative Commons Attribution-NonCommercial-NoDerivs 3.0 Unported (CC BY- NC-ND3.0) Licence (http://creativecommons.org/licenses/by-nc-nd/3.0/) which permits unrestricted noncommercial use, distribution, and reproduction in any medium, provided the original work is properly cited.

Please cite this article as: Bayram A, Eskiizmir G, Cingi C, Hanna E. Robotic Surgery in Otolaryngology-Head and Neck Surgery: Yesterday, Today and Tomorrow. ENT Updates 2020;10(2): 361-371. 